\title{
Advance Self Driving Car Using Machine Learning
}

\author{
Mohd Meraj Ahemad ${ }^{1}$, Dr.Javed Ashraf ${ }^{2} \&$ Dr.Anisur Rehman Nasir ${ }^{3}$ \\ ${ }^{I}$ M.Tech Scholar, Al-Falah University, Haryana, India. \\ ${ }^{2}$ Assistant Professor, Al-Falah University, Haryana, India. \\ ${ }^{3}$ Associate Professor, Jamia Millia Islamia (Central University), India.
}

DOI: 10.38177/ajast.2020.4307

\section{ABSTRACT}

The Autonomous car is about to enter the mass-market. The question is not about when it will happen but in which conditions, under which form or who will be the first car manufacturer to release an efficient and reliable final product. Entirely unexpected ways to deal with building up the Al frameworks for self-driving vehicles exist and most of them are horribly best in class and with extremely high equipment needs. The appropriate response presented during this paper proposes the AI fundamentally based framework to be as simple as conceivable with low equipment needs. A straight forward three layers profound, totally associated neural system was prepared to outline pictures from a forward looking QVGA camera to directing orders. Upheld an information picture the neural system should settle on one among the four offered orders (Forward, Left, Right or Stop). With least of the instructing information (250 pictures) the framework figured out how to follow the street ahead and keep in its path.The framework precisely learns essential street alternatives with exclusively the directing point in light of the fact that the contribution from the human driver. it had been near explicitly prepared to watch lines out and about. Contrasted with rather progressively confounded arrangements like express decay of the issue, similar to path identification and the board and convolutional neural systems simply like the conclusion to complete the process of learning arranged by the $\mathrm{N}$-Vidia this technique demonstrated to be amazingly solid and affordable. we will in general attempt to demonstrate that this methodology would bring about better and lower equipment necessities so making the occasion of oneself driving vehicles simpler and more financially savvy. Simple counterfeit neural system, much the same as the one gave during this paper, is sufficient for relatively muddled technique like path keeping.

Index Terms: Artificial intelligence, neural network, self-driving, autonomous vehicle, object detection, laptop vision, haarcascade, robot.

\section{Introduction}

Every year basically in US around 37,000 people are deceased in car crashes. That is a 5.8\% expansion from 2014. Human mistakes caused up to $19^{\text {th }}$ P.C of car crashes. [1] Autonomous vehicles may encourage cut back this gigantic scope of fatalities. One among the essential, most popular and most accommodating advancements is that the line recognition and path keeping. It began creating in Eighties and to the present day it's despite everything being improved. The desire of an individual to broaden the security of vehicles on street has precious stone rectifier to the occasion of different frameworks that are authorized into vehicles. Each new framework needs an extra numerical representation of the information to make determinations appropriately. Including new frameworks into the vehicles exponentially complicates the scientific model, hence the goals is a couple of sensibly a framework, that per human guidelines, is savvy for example reenacts driving as though it were a genuine driver [2]. That is anyway the idea of misuse the engineered insight was created. As software engineering advances, along these lines do its sub-branches and one in everything about preeminent fundamental branches is self-governing vehicles, which is inseparable from the pc vision. The inspiration driving this innovation is to style a framework which may do guiding, braking and quick without anyone else. During this assignment, PC vision will encourage the framework locate and build up objects, while elective calculations do the decision making [2,3].

\section{Related Work}

There are many anticipated arrangements, various they are numerical methodology, neural systems, fortification learning, convolutional neural systems, Q learning. Two most significant arrangements are numerical methodology and CNNs. every ways have their executives and cons. This paper presents a simple, brisk and viable way to deal 
with handle this drawback. Mathematical approach needs explicit disintegration of the issue. This technique will utilize monocular or sound system vision. The greater part of those systems was that spend significant time in identification of path markers on the fundamental street, which are nearly easy to take note. For the most part, it needs benchmark to be even, for example skyline inside the picture is corresponding to $\mathrm{x}$ pivot. Lines must be sufficiently thick and in a very type of parallelogram (or rough). When the street limits are distinguished the situation of auto will be determined, with pre-determined action data for the camera. [3, 4,5] With incredible vehicle position and through cutting edge framework and unadulterated science figuring's, required controlling edge will be determined. Diverse comparable strategies need level street, incredible separation, optical focus, pitch point, yaw edge and stature higher than ground before performing expressions scientific changes. [6]Another approach is by utilizing convolutional Neural Systems (CNNs). For this methodology important issue is to assemble substantial information. It is done, by sparing orders given by human driver and pictures from on board cameras. CNNs have changed example acknowledgment [7]. They're fit for gaining choices precisely from past information. On account of CNNs significant level of value it needs high completion equipment to run. A large portion of the arrangements of this sort are utilizing different realistic procedure units (GPUs) or some devoted equipment like N-Vidia DRIVE post trade, every one of that enormously quicken learning rate also on the grounds that the execution of a prepared system. Of these arrangements accompanied normal defects like: tremendous, clumsy, unpredictable, overrated and not frightfully power conservative equipment. [8] One epic answer misuse CNNs is in this manner known as end to complete the process of learning created by N-Vidia. With nearly contact of instructing information from human driver, framework took in the best approach to drive a car [7]. Similarly to the appropriate response gave during this paper. One gigantic blemish of this methodology is that the utilization of the N-Vidia Dev box and Drive post trade, with consolidated cost in a huge number of bucks. The arrangement anticipated during this paper means to create an a ton of conservative execution of a self-governing driving AI rule. Abuse this methodology; it's possible to make self-driving vehicle while not an express significant level numerical demonstrating and examination of the issue. Additionally, it needn't bother with eager for power, amazing and overrated equipment for execution. It's fit to be prepared misuse horrendously contact of past information, because of its effortlessness.

\section{System Overview}

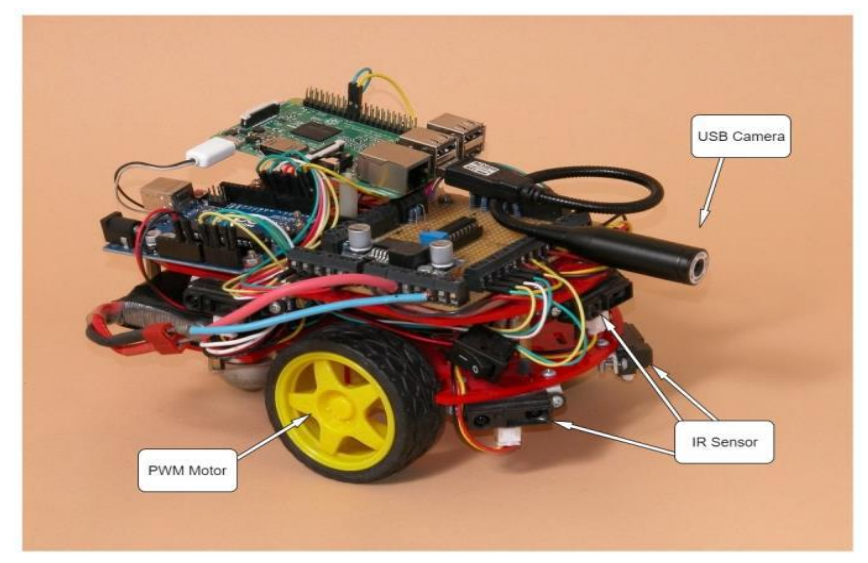

Fig.1 Overview of the robot 
The machine utilized right now uniquely made, constrained by Raspberry Pi and Arduino PCs. registering that controlled the robot was dead on a different PC. Correspondence between the 2 was done over the TCP/IP network [8,9]. Robot was driven by 2 steam-fueled haggles single detached wheel. It had 5 IR nearness sensors, 3 looking ahead and 2 on the borders and a forward looking camera. The IR sensors were utilized in impediment recognition though the camera was utilized in sign and traffic signal location robot is appeared inside the Fig. 1.

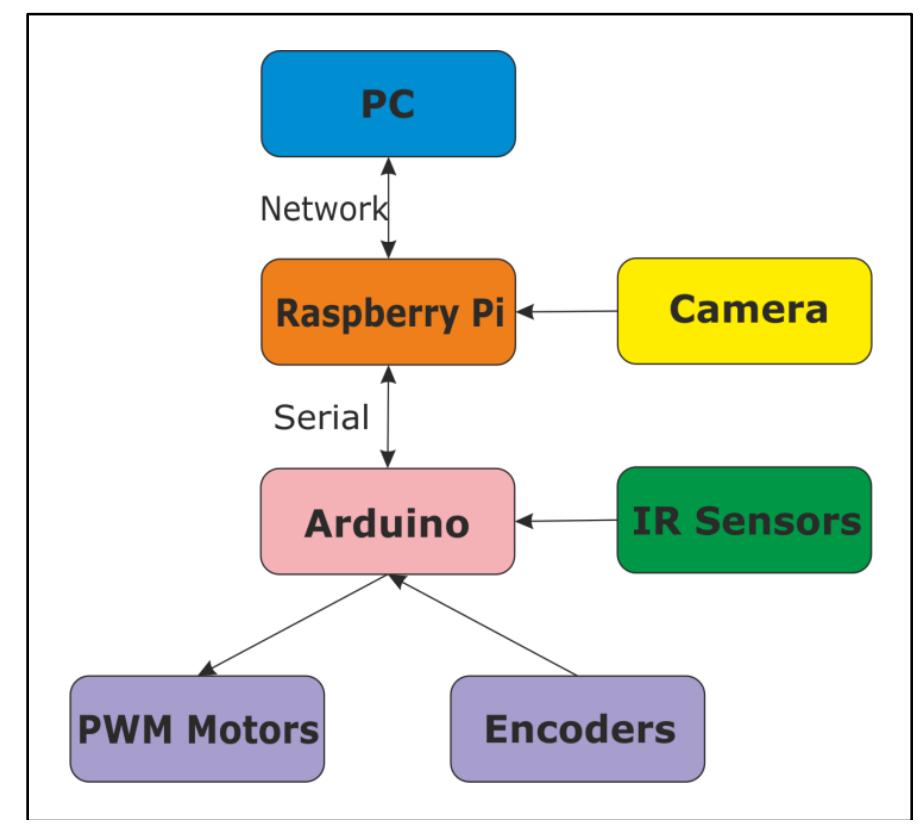

Fig.2 Connections of the electrical components of the system

All modules speak with each other misuse very much sketched out conventions, this licenses basic updating and scaling once required. Framework is expected to deal with various vehicles, different clients and numerous AI operators. This can be accomplished by arranging the framework to be causative and keeping in mind that not memory, allowing vehicles to be constrained by numerous examples of the AI specialist simultaneously comparably as endorsing the use of the heap balancer between the occurrences of the AI operator for progressively reasonable quantifiability [10].

\section{Data Collection}

Preparing information was gathered by physically driving the vehicle on a model street. Other than the normal street design any place street (black-top) is dark with white lines, the vehicle is furthermore prepared and tried with the darker street, with white lines. Training data was gathered in manual mode abuse pictures from the forward looking camera exclusively [11].

Gathered data is keep as an attempt of qualities. Manus parameter is order that was sent to the vehicle (bearing of development) and furthermore the second might be a video outline at the moment the exact order was given. Preparing information was gathered by physically driving the vehicle on a model street $[12,13]$.

Other than the normal street design any place street (black-top) is dark with white lines, the vehicle is furthermore prepared and tried with the darker street, with white lines. 


\section{Artificial Intelligence System}

Man-made intelligence is responsible for issue the orders to the lower level module (vehicle). It's structured as numerous state machines. Figuring on this state, entirely unexpected AI equation is the system is basically 3 layers profound, the info layer (picture from the camera), one concealed layer and in this manner the yield layer (directing order). It's a simple completely associated organize.

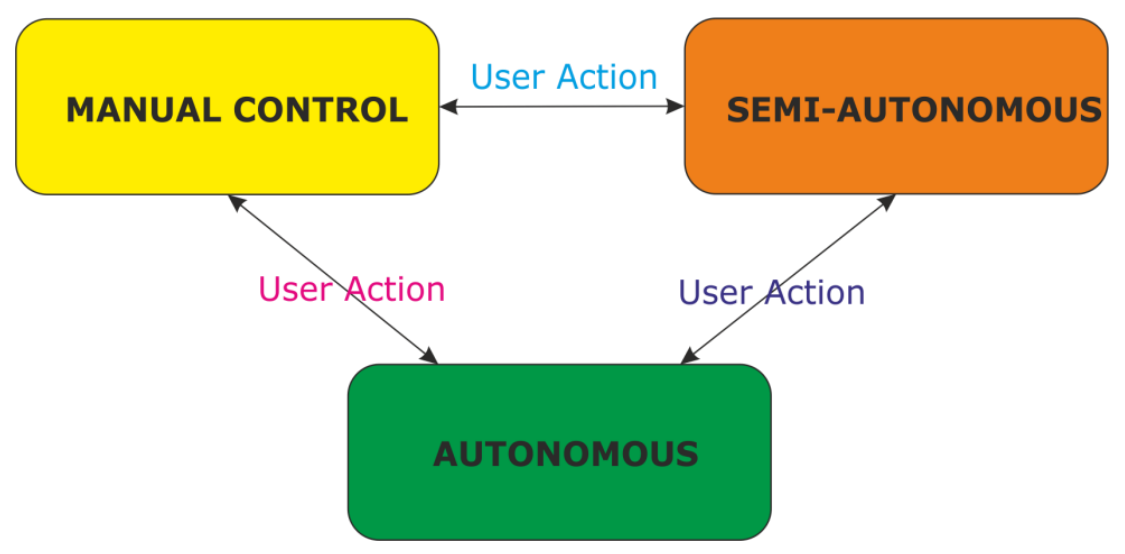

Fig.3 Autonomous vehicle operation modes

The yield from the system is one among the feasible four choices (three bearings: forward, forward-left, forward-right and a stop command) [14].This makes future updates and a change of explicit calculations simpler to implement. Implementation spoke to during this paper has 3 very surprising methods of activity. As appeared inside the Fig.4. The vehicle begins inside the "Manual control" mode. From that point it will be become elective modes upheld a client input.

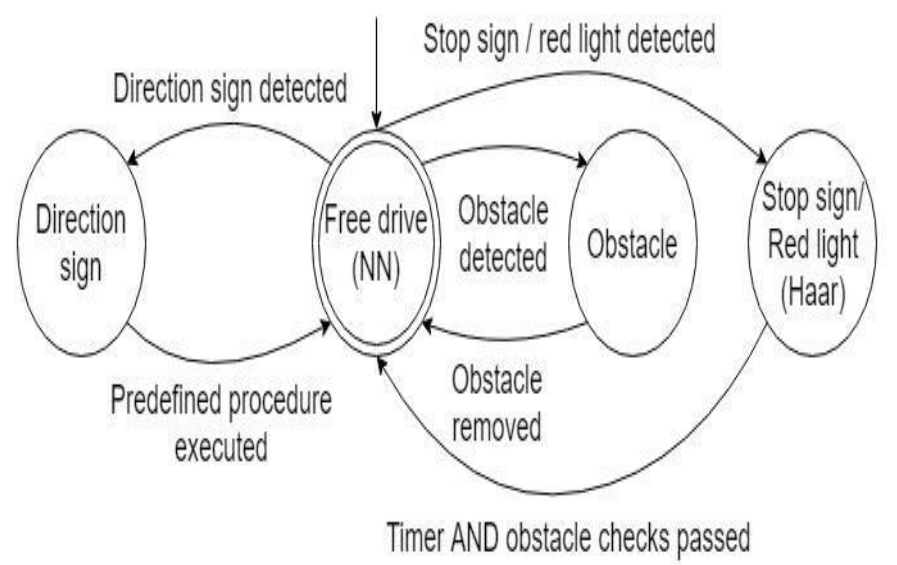

Fig.4 Autonomous mode finite state machine

In "Semi-self-sufficient" mode the state machine is simple. There are just two states are conceivable .The most interesting is that the state machine in "Self-governing" mode, for example autopilot, that is appeared in Fig. 5. It's of four states. Once more, "Free drive" is start and end state. During this state ANN has the administration of the vehicle. Seeing is being determined on the info edges and sensors are never-endingly checked for impediments. 
From this express it's capability to progress to the next state wagering on the identified article or the data from IR sensors. However, if the bearing sign is distinguished, the state machine changes into "Course sign" state. During this express, the vehicle starting should check if there's approaching traffic on the decussate street [14,15]. On the off chance that the convergence is obvious, the vehicle executes order for example turns inside the ideal bearing. At the point when this technique, the state machine is changed into "Free drive" mode and along these lines the administration is offered back to the ANN.

\section{Sign Detection}

Traffic sign identification and arrangement is unmistakably fundamental for self-driving vehicles. The Open CV library gives instruments required to this during a kind of a coach and an identifier. It had been resolved to actualize exclusively classifiers for stop sign, traffic lights and heading signs (left, right, forward and switch back). Since, exploitation this methodology, each item wants its own classifier and since scaling is scarcely the topic of getting the worthy training information, it had been resolved this can be sufficient for the confirmation of conception. The conduct of the vehicle once the sign is distinguished is hard-coded. For all the signs and furthermore the stoplight the hole to the thing is estimated.

\section{Conclusion}

One admonition of any AI framework is that the capricious conduct. Anyway during this trial, it totally was incontestable that the occasion of those sorts of frameworks will be simple, especially for the noncritical frameworks. For this present reality application, further testing would be required, yet as some further wellbeing conventions. Anyway the entirety of this could be accomplishable with the framework that was incontestable. All of this was accomplished utilizing at least information and incredibly unobtrusive equipment. That was sufficient to mentor the framework to drive in different conditions, from day to evening, from urban roads to roadways, independent of the amount of various members in rush hour gridlock and change in accordance with all the transit regulations.

\section{Reference}

[1] U.S. Department of Transportation's National Highway Traffic Safety Administration.

(2016). Retrieved from United States Department of Transportation: crashstats.nhtsa.dot.gov/Api/Public/Publication/812456

[2] Tan, B., Xu, N., \& Kong, a. B. (2018). Autonomous Driving in Reality with Reinforcement Learning and Image. arXiv preprint arXiv:1801.05299. Retrieved from https://arxiv.org/pdf/1801.05299

[3] Massimo, B., \& Broggi, A. (1996). Real-time lane and obstacle detection on the GOLD system. Intelligent Vehicles Symposium, 1996., Proceedings of the 1996 IEEE, 213-218.

[4] Bertozzi, M., Broggi, A., Conte, G., \& Fascioli, A. (1997). Obstacle and lane detection on ARGO. Intelligent Transportation System, 1997. ITSC'97., IEEE Conference on. IEEE, 1010-1015.

[5] Wang, H., \& Chen, Q. (2016). Real-time lane detection in various conditions and night cases. Intelligent Transportation Systems Conference, ITSC '06. IEEE, 1226-1231. 
[6] Aly, M. (2008). Real time detection of lane markers in urban streets. Intelligent Vehicles Symposium, IEEE, $7-12$.

[7] Bojarski, M., Del Testa, D., Dworakowski, D., Firner, B., Flepp, B., Goyal, P., Jackel, L.D., Monfort, M., Muller, U., Zhang, J. and Zhang, X. (2016). End to end learning for self-driving cars. arXiv preprint arXiv:1604.07316.

[8] Krizhevsky, A., Sutskever, I. and Hinton, G.E. (2012). Image Net Classification with Deep Convolutional. Advances in neural information processing systems, 1097-1105.

[9] Y. LeCun, B. Boser, J. S. Denker, D. Henderson, R. E. Howard, W. Hubbard, and L. D. Jackel. Backpropagation

[10] Alex Krizhevsky, Ilya Sutskever, and Geoffrey E. Hinton. Imagenet classification with deep convolutional neural networks. In F. Pereira, C. J. C. Burges, L. Bottou, and K. Q. Weinberger, editors, Advances in Neural Information Processing Systems 25, pages 1097-1105. Curran Associates, Inc., 2012.

[11] L. D. Jackel, D. Sharman, Stenard C. E., Strom B. I., , and D Zuckert. Optical character recognition for self-service banking. AT\&T Technical Journal, 74(1):16-24, 1995.

[12] Large scale visual recognition challenge (ILSVRC). URL: http://www.image-net.org/ challenges/LSVRC.

[13] Net-Scale Technologies, Inc. Autonomous off-road vehicle control using end-to- end learning, July 2004.Final technical report.

URL: http://net-scale.com/doc/net-scale-dave-report.pdf.

[14] Dean A. Pomerleau. ALVINN, an autonomous land vehicle in a neural network. Carnegie Mellon University, 1989. URL: http://repository.cmu.edu/cgi/viewcontent.

[15] Wikipedia.org. DARPA LAGR program. http://en.wikipedia.org/wiki. 